\title{
Correction to: Multi-server queueing systems with multiple priority classes
}

\author{
Mor Harchol-Balter ${ }^{1} \cdot$ Takayuki Osogami $^{2}$ - Alan Scheller-Wolf ${ }^{3}$. \\ Adam Wierman ${ }^{4}$
}

Received: 15 July 2021 / Revised: 15 July 2021 / Accepted: 15 July 2021 /

Published online: 27 September 2021

(c) Springer Science+Business Media, LLC, part of Springer Nature 2021

\section{Correction to: Queueing Syst https://doi.org/10.1007/s11134-005-2898-7}

We correct the expressions of the matrix $\mathbf{B}^{(\ell)}$ on page 340 and the matrix $\mathbf{L}^{(\ell)}$ on page 341 in [1]. Specifically, the following are the corrected expressions of these matrices:

$$
\mathbf{B}^{(\ell)}=\mu_{L}\left(\begin{array}{llll}
\min (2, \ell) & & & \\
& 1 & & \\
& & 1 & \\
& & & 0
\end{array}\right)
$$

The original article can be found online at https://doi.org/10.1007/s11134-005-2898-7.

\Takayuki Osogami osogami@jp.ibm.com

Mor Harchol-Balter harchol@cs.cmu.edu

Alan Scheller-Wolf awolf@andrew.cmu.edu

Adam Wierman adamw@caltech.edu

1 Department of Computer Science, Carnegie Mellon University, 5000 Forbes Ave., Pittsburgh, PA 15213, USA

2 Research, IBM, 19-21 Nihonbashi Hakozaki-cho, Chuo-ku, Tokyo 103-8510, Japan

3 Tepper School of Business, Carnegie Mellon University, 5000 Forbes Ave., Pittsburgh, PA 15213, USA

4 Computing and Mathematical Sciences, California Institute of Technology, 1200 E. California Blvd, Pasadena, CA 91125, USA 


$$
\mathbf{L}^{(\ell)}=\left(\begin{array}{cc|c|c|c|c|c|c}
-\sigma_{1} & \lambda_{M} & \lambda_{H} & & & & \\
\mu_{M}-\sigma_{2} & \lambda_{M} \mathbf{p}^{(2 M, M)} & \lambda_{M} \mathbf{p}^{(2 M, H)} & \lambda_{H} \mathbf{p}^{(M H, M)} & \lambda_{H} \mathbf{p}^{(M H, H)} & & \\
\mu_{H} & -\sigma_{3} & & & \lambda_{M} \mathbf{p}^{(M H, M)} & \lambda_{M} \mathbf{p}^{(M H, H)} & \lambda_{H} \mathbf{p}^{(2 H, M)} & \lambda_{H} \mathbf{p}^{(2 H, H)} \\
\hline \mathbf{t}^{(1)} & \mathbf{T}^{(1)} & & & & & \\
\hline \mathbf{t}^{(2)} & & \mathbf{T}^{(2)} & & & & \\
\hline \mathbf{t}^{(3)} & & & \mathbf{T}^{(3)} & & & \\
\hline \mathbf{t}^{(4)} & & & & \mathbf{T}^{(4)} & & \\
\hline \mathbf{t}^{(5)} & & & & & \mathbf{T}^{(5)} & \\
\hline \mathbf{t}^{(6)} & & & & & & \mathbf{T}^{(6)}
\end{array}\right)
$$

for all $\ell \geq 0$, where the definitions of the notation in the matrices are unchanged from [1] except the zero matrix $\mathbf{0}$ in (1), whose size needs to be corrected to $12 \times 12$.

These matrices represent the transitions shown in the left panel of Figure 3 in [1]. The transition from state $(1 \mathrm{H}, 0 \mathrm{M}, u \mathrm{~L})$ to $(1 \mathrm{H}, 0 \mathrm{M},(u-1) \mathrm{L})$, namely the third diagonal element of $\mathbf{B}^{(\ell)}$, was missing in the original expression. The transition rates from $(0 \mathrm{H}, 1 \mathrm{M}, u \mathrm{~L})$ to two states labeled with $(1 \mathrm{H}, 1 \mathrm{M}, u \mathrm{~L})$, namely the $(2, k)$ element of $\mathbf{L}^{(\ell)}$ for $8 \leq k \leq 11$, are $\lambda_{H} \mathbf{p}^{(M H, M)}$ and $\lambda_{H} \mathbf{p}^{(M H, H)}$, but erroneously were $\lambda_{M} \mathbf{p}^{(M H, M)}$ and $\lambda_{M} \overline{\mathbf{p}}^{(M H, H)}$ in the original expression. Likewise, the transition rates from $(1 \mathrm{H}, 0 \mathrm{M}, u \mathrm{~L})$ to $(1 \mathrm{H}, 1 \mathrm{M}, u \mathrm{~L})$, namely the $(3, k)$ element of $\mathbf{L}^{(\ell)}$ for $8 \leq k \leq 11$, are $\lambda_{M} \mathbf{p}^{(M H, M)}$ and $\lambda_{M} \mathbf{p}^{(M H, H)}$, but were $\lambda_{H} \mathbf{p}^{(M H, M)}$ and $\lambda_{H} \mathbf{p}^{(M H, H)}$ in the original expression.

Acknowledgements We thank Dr. Yee Lam Elim Thompson for pointing out the errors.

\section{Reference}

1. Harchol-Balter, M., Osogami, T., Scheller-Wolf, A., Wierman, A.: Multi-server queueing systems with multiple priority classes. Queueing Syst. 51, 331-360 (2005). https://doi.org/10.1007/s11134-0052898-7

Publisher's Note Springer Nature remains neutral with regard to jurisdictional claims in published maps and institutional affiliations. 